\title{
A Descriptive Analysis of Clinical Articles Published in the Last 50 Years in the Dental Literature
}

\author{
Zuhair S Natto ${ }^{1}$, Ahmad Aljehani ${ }^{2}$, Anfal Sarhan ${ }^{3}$, Elaf Nawawi ${ }^{4}$, Hanan Abdullatef ${ }^{5}$, Lina Samarkandi ${ }^{6}$, Maryam Nasser $^{7}$, \\ Rawan Badri ${ }^{8}$, Rufaida Quqandi ${ }^{9}$, Sara Waheeb ${ }^{10}$, Sarah Aljahdali ${ }^{11}$, Yasser Merdad ${ }^{12}$
}

\begin{abstract}
Aims: This article describes the methodologies used in the dental literature and described how these approaches have changed over time Materials and methods: Thirty-three ISI peer-reviewed journals were included in the analyses. Data were extracted independently by 11 investigators and in duplicate. Any differences in the results were resolved via discussion or by a third reviewer when necessary. Data were collected regarding the methodology used in the article, and dental specialty related to different study designs. In the case in which more than one study design or specialty was reported, reviewers were trained to identify the main methodology/specialty.

Results: The majority (36.96\%) used a case report (CR) as the primary methodology, followed by a clinical trial (CT) (18.21\%) or randomized CT (15.11\%). The least used methodologies included a cohort (COH) study $(6.07 \%)$ or a systematic review (SA)/meta-analysis (MA) (6.73\%). Periodontology published the highest number of case controls (CCs) (46.8\%), randomized CTs (RCTs) (29.9\%), cross-sectional (CS) studies (26.0\%), SRs/MAs (19.8\%), and CTs (17.1\%). Oral and maxillofacial surgery published the highest number of CRs/case series (54.5\%) and COH studies (30.5\%), whereas operative dentistry published the lowest number of CRs/case series (0.7\%), CCs (2.9\%), and SRs/MAs (2.3\%). CRs/case series retain the highest number of publications across all time points in the dental literature overall.

Conclusion: Our results indicate an improvement in the types of research and the pyramid of evidence, which will help in applying evidencebased dentistry (EBD) in clinical decision-making.

Clinical significance: Types of studies used in the dental field are not yet investigated. Thus, little is known about the common study design types in dental literature. This can affect the decision made regarding technique, risk factors, prevention, or treatment.

Keywords: Case controls, Case reports, Clinical trials, Cohort designs, Cross-sectional studies, Dental, Randomized clinical trials, RCTs, Type of articles. The Journal of Contemporary Dental Practice (2019): 10.5005/jp-journals-10024-2613
\end{abstract}

\section{BACKGROUND}

EBD focuses on answering clinical queries using the best available evidence and is involved in every part in dentistry. In recent decades, EBD was the standard for decision-making and was adopted in all dental specialties; ${ }^{1}$ consequently, the quantity and the quality of all studies have increased throughout the dental literature. ${ }^{1,2}$ Such improvements help clinicians to make the best decisions based on objective assessments of various treatment options. ${ }^{1,2}$

In EBD, it is important to use the highest quality evidence available in clinical decision-making. SRs and MAs of randomized control trials consider the highest level of available information and that which is in the top of the pyramid of evidence. ${ }^{3-6}$ Nowadays, dental practitioners are becoming increasingly dependent on SAs and MAs, to obtain valid and reliable clinical results, which are highly valuable to finalize decisions in clinical practice. ${ }^{3,5-8}$

One way of assessing the influence of EBD within all dental specialties is to evaluate the types of articles published within each specialization and evaluate the hierarchy of evidence used. Moreover, it is useful to investigate how such approaches change over time. Hence, we designed a study to evaluate articles published in the dental literature over the past 50 years. Additionally, we examined whether the type of research design has changed over the established period, parallel to the prevalence of EBD.

\section{Materials and Methods}

\section{Search Strategy and Inclusion Criteria}

All 33 Institute for Scientific Information (ISI) peer-reviewed journals published in the past 50 years were included in the analyses from
${ }^{1}$ Department of Dental Public Health, School of Dentistry, King Abdulaziz University, Jeddah, Kingdom of Saudi Arabia; Department of Periodontology, School of Dental Medicine, Tufts University, Boston, Massachusetts, USA; Department of Oral Health Policy and Epidemiology, School of Dental Medicine, Harvard University, Boston, Massachusetts, USA

${ }^{2} \mathrm{SHO}$ General Dentistry, King Fahd Armed Forces Hospital, Jeddah, Kingdom of Saudi Arabia

${ }^{3,6} \mathrm{Al}$-Farabi College for Nursing and Dentistry, Jeddah, Kingdom of Saudi Arabia

$4,7,10,11$ Ibn Sina National College for Medical Studies, Jeddah, Kingdom of Saudi Arabia

${ }^{5}$ Private Clinic, Jeddah, Kingdom of Saudi Arabia

${ }^{8}$ Department of Periodontology, School of Dentistry, King Abdulaziz University, Jeddah, Kingdom of Saudi Arabia

${ }^{9}$ Department of Prosthodontics, School of Dentistry, King Abdulaziz University, Jeddah, Kingdom of Saudi Arabia

${ }^{12}$ Department of Restorative Dentistry, School of Dentistry, King Abdulaziz University, Jeddah, Kingdom of Saudi Arabia

Corresponding Author: Zuhair S Natto, Department of Dental Public Health, School of Dentistry, King Abdulaziz University, Jeddah, Kingdom of Saudi Arabia, Phone: +96 6126943899, e-mail: znatto@kau.edu.sa

How to cite this article: Natto ZS, Aljehani A, et al. A Descriptive Analysis of Clinical Articles Published in the Last 50 Years in the Dental Literature. J Contemp Dent Pract 2019;20(7):867-872.

Source of support: Nil

Conflict of interest: None

() The Author(s). 2019 Open Access This article is distributed under the terms of the Creative Commons Attribution 4.0 International License (https://creativecommons. org/licenses/by-nc/4.0/), which permits unrestricted use, distribution, and non-commercial reproduction in any medium, provided you give appropriate credit to the original author(s) and the source, provide a link to the Creative Commons license, and indicate if changes were made. The Creative Commons Public Domain Dedication waiver (http://creativecommons.org/publicdomain/zero/1.0/) applies to the data made available in this article, unless otherwise stated. 
1967 through 2017 (Table 1). Some study designs published in the dental literature were not part of the analyses, such as editorial comments, literature reviews, book reviews, lab/animal studies, and published proceedings from professional meetings and conferences. In all, 37,868 articles were included in the final analysis (Flowchart 1). Using a search strategy by looking under each journal, 11 investigators (two in each article) - ZN with AA, AS, EN, HA, LS, MN, RB, RQ, SW, SA, or YM-screened MEDLINE, Scopus, Web of Science, Embase, and Cochrane Central Register of Controlled Trials, Cochrane Central SR, and meta-analysis. Additionally, we screened Internet search engines, such as Google Scholar.

\section{Data Extraction and Reliability}

We extracted data independently in duplicate. Any differences were resolved via discussion or by a third reviewer in the same group who was involved when necessary. Data were collected regarding the methodology used in the article (SAs/MAs; CTs; CTs; RCTs; CS studies; $\mathrm{CCs}$; or $\mathrm{COH}$ designs), and dental specialty related to different study designs. In the case in which more than one study design or specialty was reported, reviewers were trained to identify the main methodology/specialty. Interexaminer agreements were calculated using a Cohen k coefficient, which was above 0.8 . The Fischer exact test was conducted to assess if there was an association between the type of studies and the dental specialty.

\section{Results}

\section{Methodology Used in Published Articles}

We categorized types of studies based on the main methodology used in the study. The majority (36.96\%) used a CT as the primary methodology, followed by a CT (18.21\%) or RCT (15.11\%). The least used methodologies included a $\mathrm{COH}(6.07 \%)$ or a CS study $(6.71 \%)$ (Table 2).
Table 1: List of included journals

\begin{tabular}{ll}
\hline Journal name & Journal name \\
\hline - Oral Oncology & - Clinical Oral Implants Research \\
- International Journal of Oral & - Journal of Dentistry \\
Science & \\
- Journal of Oral Implantology & - The International Journal of \\
& Prosthodontics \\
- Journal of Oral and & - European Journal of \\
Maxillofacial Surgery & Orthodontics \\
- International Journal of Oral & - Oral and Maxillofacial Surgery \\
and Maxillofacial Surgery & Clinics of North America \\
- Journal of Craniofacial Surgery & - The Journal of Adhesive \\
& Dentistry \\
- The Angle Orthodontist & - Journal of Dental Research \\
- Journal of Pediatric Dentistry & - Dental Materials Journal \\
- International Journal of & - Journal of Oral Pathology and \\
Paediatric Dentistry & Medicine \\
- Caries Research & - Journal of Prosthetic Dentistry \\
- American Journal of Orthodon- & - International Journal of Oral \\
tics and Dentofacial Orthopedics & and Maxillofacial Implants \\
- Community Dentistry and Oral & - Journal of Prosthodontic \\
Epidemiology & Research \\
- Journal of Periodontal & - Journal of Oral and Facial Pain \\
Research & and Headache \\
- Operative Dentistry & - Journal of Endodontics \\
- International Endodontic & - European Journal Of Oral \\
Journal & Implantology \\
- Clinical Implant Dentistry and & - Journal of Clinical \\
Related Research & Periodontology \\
- Journal of Periodontology & - \\
\hline & \\
\hline
\end{tabular}

Flowchart 1: The search strategy

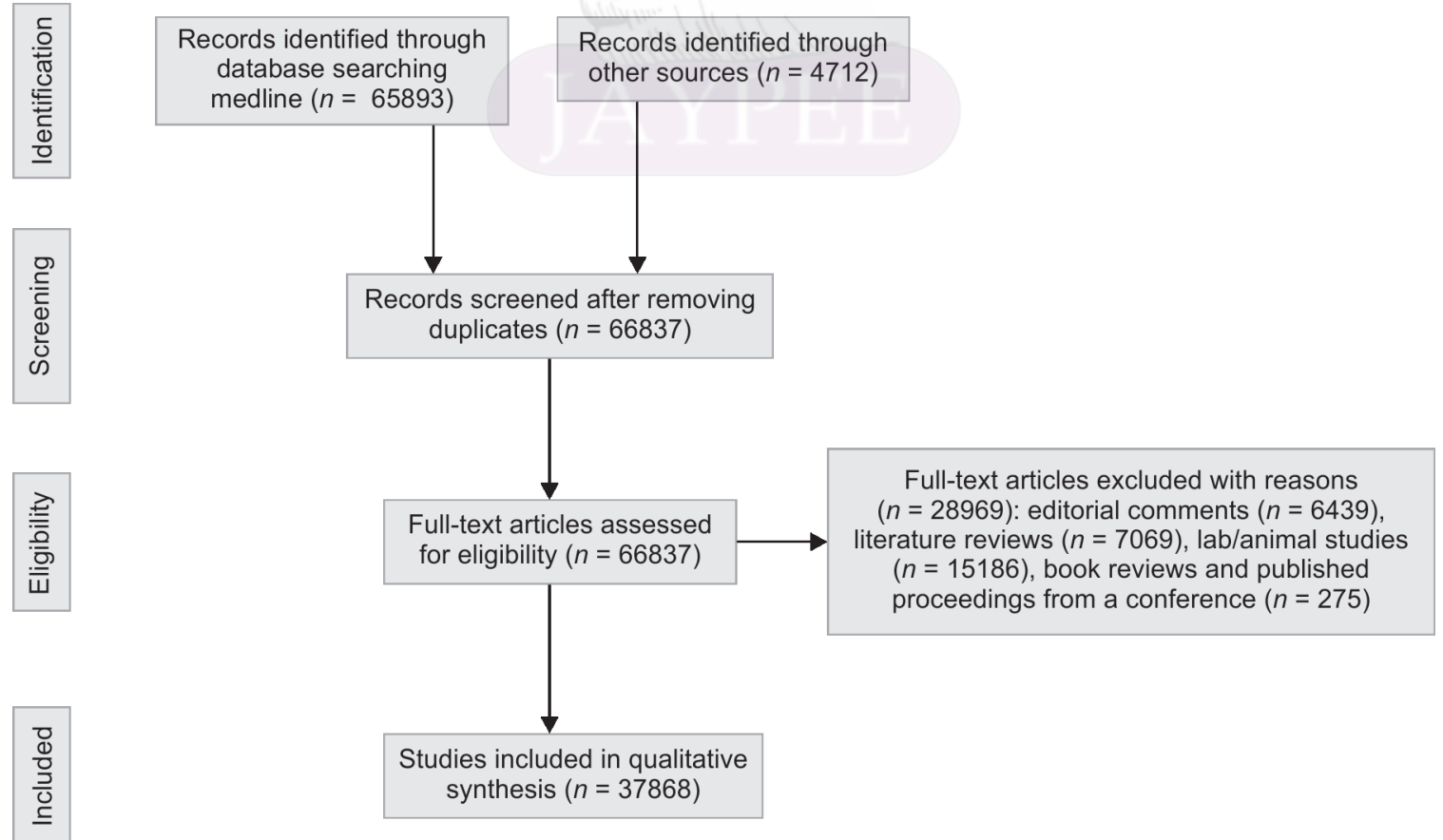


Table 2: Frequency and percentage of publication types in all dental specialties

\begin{tabular}{lll}
\hline Publication type & \# of articles & $\%$ \\
\hline Systematic review/meta-analysis & 2,550 & 6.73 \\
Clinical trial & 6,894 & 18.21 \\
Case report & 13,995 & 36.96 \\
Randomized clinical trial & 5,721 & 15.11 \\
Cross-sectional & 2,540 & 6.71 \\
Case control & 3,871 & 10.22 \\
Cohort & 2,297 & 6.07 \\
Total & 37,868 & 100 \\
\hline
\end{tabular}

\section{Methodology Used in Each Dental Specialty}

The articles within each specialty were further classified based on the type of study. Findings reveal that periodontology published the highest number of CCs (46.8\%), RCTs (29.9\%), CS studies (26.0\%), SRs/MAs (19.8\%), and CTs (17.1\%), whereas oral and maxillofacial surgery published the highest number of CTs/case series (54.5\%) and COH studies (30.5\%) (Table 3).
Operative dentistry published the lowest number of CTs/case series $(0.7 \%)$, CCs (2.9\%), and SRs/MAs (2.3\%); oral medicine published the lowest number of RCTs (1.6\%), CTs (2.5\%), and CS studies (4.3\%) (Table 3). Overall $p$ value was $<0.05$ which means that there is an association between the type of study and the dental specialty.

\section{Types of Publications Over Time}

Case report and case series remain the most frequently utilized methodology across all points times in the dental literature overall, followed by clinical trials and RCTs (Fig. 1). Each dental specialty has its own pattern. In oral medicine, for example, there is increasing in number of case controls, cohort studies, and SRs/MAs, as well as a decreasing number of case reports (Fig. 2). The areas of periodontology (Fig. 2C) and implant dentistry (Fig. 3) have the same trends in their publications using RCTs and clinical trial, both of which showed increased publications in recent decades. The areas of oral maxillofacial surgery (Fig. 2D) and prosthodontic (Fig. 2F) still demonstrate a high trend of case report publications, while operative dentistry (Fig. 2A) and endodontics (Fig. 2E) demonstrate a high trend toward using and publishing clinical trials and RCTs in recent decades.

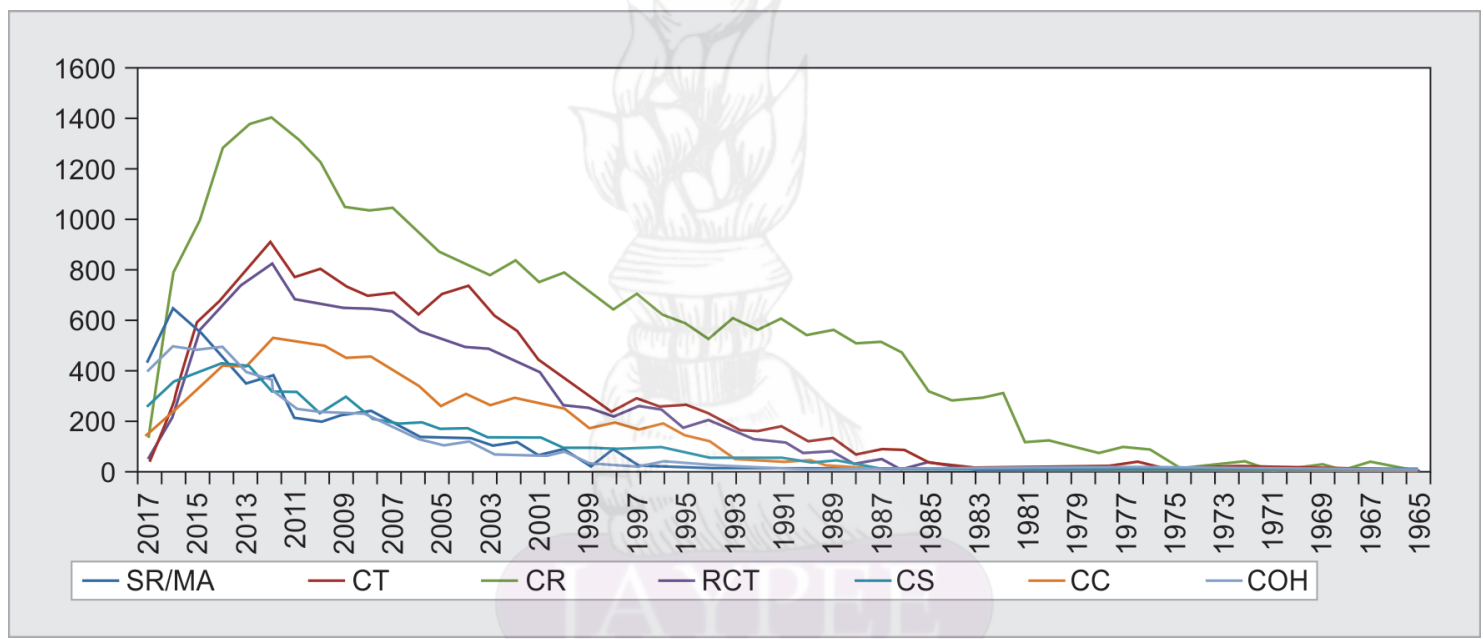

Fig. 1: Publication pattern in all dental literature over 50 years (SR/MA, systematic review/meta analysis; $C T$, clinical trial; $C R$, case report; $R C T$, randomized clinical trial; $\mathrm{CS}$, cross-sectional; $\mathrm{CC}$, case control; $\mathrm{COH}$, cohort)

Table 3: Frequency and percentage of publication types by different dental fields

\begin{tabular}{|c|c|c|c|c|c|c|c|}
\hline & $\begin{array}{l}\text { Systematic } \\
\text { review/meta } \\
\text { analysis } \\
(n=2,550)\end{array}$ & $\begin{array}{l}\text { Clinical trial } \\
(n=6,894)\end{array}$ & $\begin{array}{l}\text { Case report } \\
(n=13,995)\end{array}$ & $\begin{array}{l}\text { Randomized } \\
\text { clinical trial } \\
(n=5,721)\end{array}$ & $\begin{array}{l}\text { Cross } \\
\text { sectional } \\
(n=2,540)\end{array}$ & $\begin{array}{l}\text { Case control } \\
(n=3,871)\end{array}$ & $\begin{array}{l}\text { Cohort } \\
(n=2,297)\end{array}$ \\
\hline Oral Medicine & $126(4.94)$ & $170(2.47)$ & $483(3.45)$ & $91(1.59)$ & $108(4.25)$ & $512(13.23)$ & $294(12.80)$ \\
\hline Periodontics & $504(19.76)$ & $1,178(17.09)$ & $909(6.50)$ & $1,711(29.91)$ & $661(26.02)$ & $1,188(46.77)$ & 449 (19.55) \\
\hline Implant Dentistry & $461(18.08)$ & $1,058(15.35)$ & $793(5.67)$ & $613(10.71)$ & $184(7.24)$ & $206(8.11)$ & $299(13.02)$ \\
\hline Endodontics & $170(6.67)$ & $717(10.40)$ & $1,066(7.62)$ & $642(11.22)$ & $232(9.13)$ & $153(6.02)$ & 89 (3.87) \\
\hline Operative Dentistry & $59(2.31)$ & $925(13.42)$ & $97(0.69)$ & $776(13.56)$ & $211(8.31)$ & $74(2.91)$ & $101(4.40)$ \\
\hline Prosthodontics & $202(7.92)$ & $510(7.40)$ & $994(7.10)$ & $311(5.44)$ & $146(5.75)$ & $138(5.43)$ & $82(3.57)$ \\
\hline Pediatric Dentistry & $204(8.00)$ & $335(4.86)$ & $795(5.68)$ & $289(5.05)$ & $237(9.33)$ & $207(8.15)$ & $120(5.22)$ \\
\hline Orthodontics & $339(13.29)$ & 909 (13.19) & $1,237(8.84)$ & $621(10.85)$ & $435(17.13)$ & $652(25.67)$ & $162(7.05)$ \\
\hline $\begin{array}{l}\text { Oral and Maxillofacial } \\
\text { Surgery }\end{array}$ & 485 (19.02) & $1,092(15.84)$ & 7,621 (54.46) & $667(11.66)$ & $326(12.83)$ & $741(29.17)$ & $701(30.52)$ \\
\hline
\end{tabular}

Overall $p$ value is $<0.05$ 

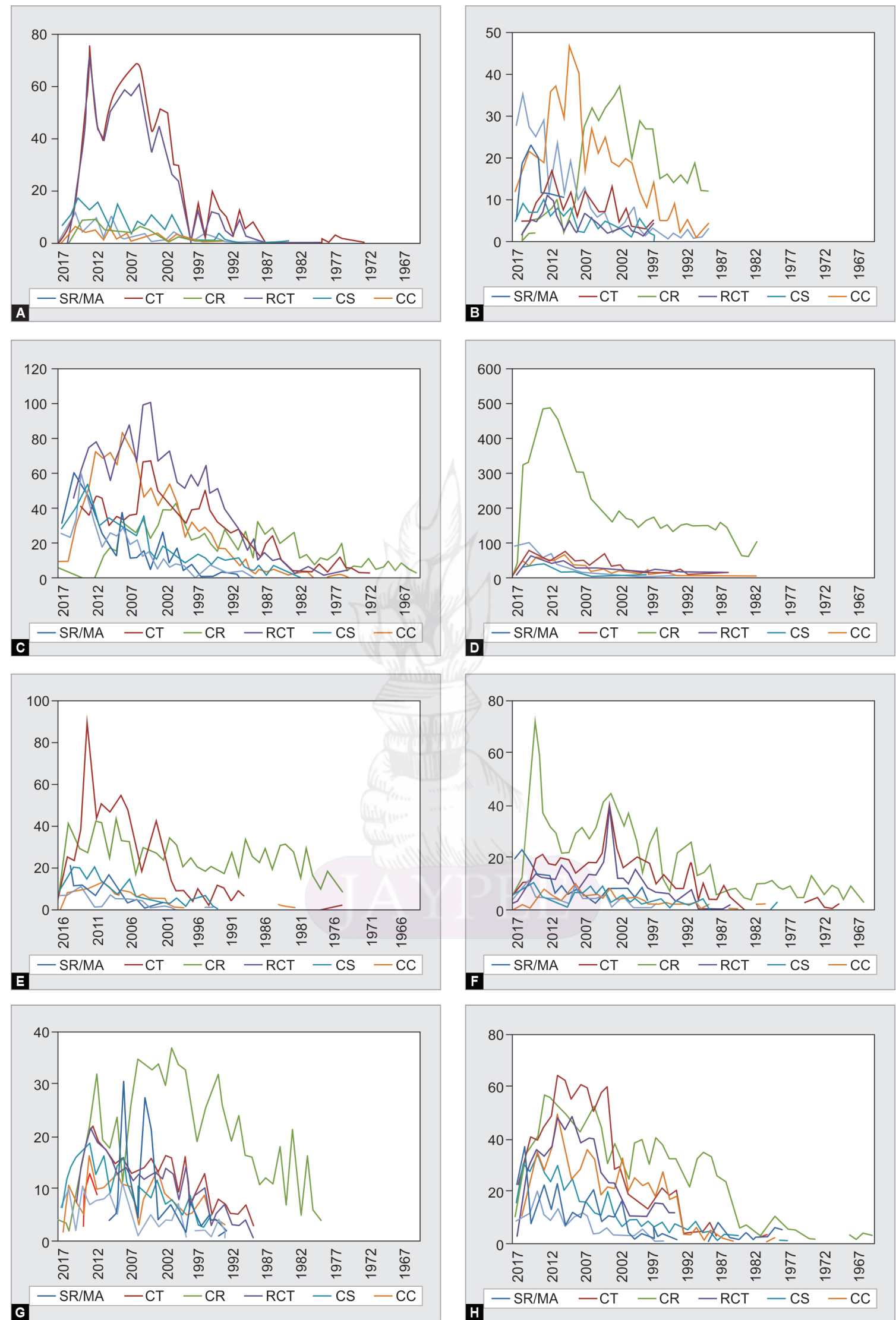

Figs $2 \mathrm{~A}$ to $\mathrm{H}$ : Publication pattern in all dental specialties over 50 years. (A) Operative dentistry; (B) Oral medicine; (C) Periodontology; (D) Oral and maxillofacial surgery; (E) Endodontics; (F) Prosthdontics; (G) Pediatric dentistry; (H) Orthodontics. (SR/MA, systematic review/meta analysis; CT, clinical trial; $\mathrm{CR}$, case report; $\mathrm{RCT}$, randomized clinical trial; $\mathrm{CS}$, cross-sectional; CC, case control; $\mathrm{COH}$, cohort) 


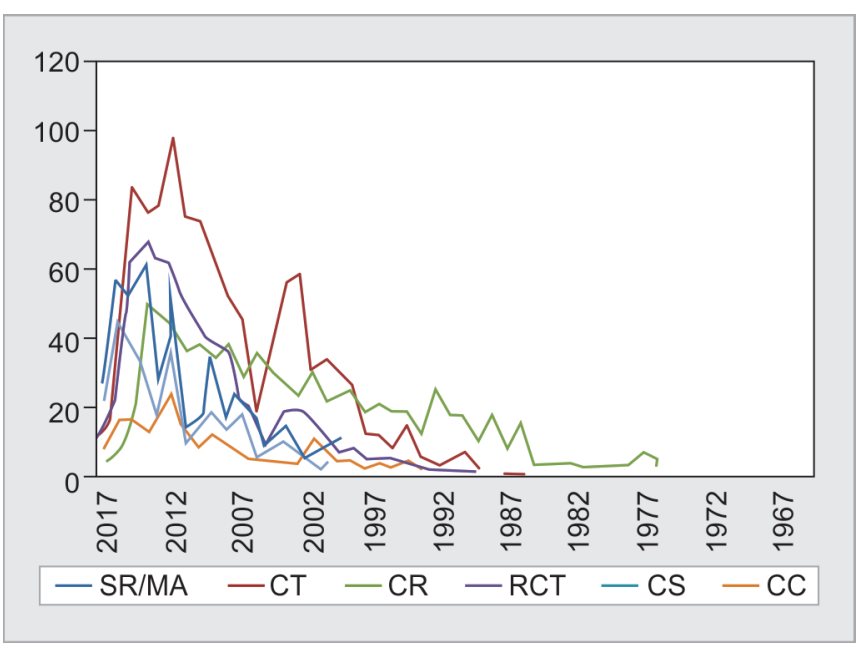

Fig. 3: Publication pattern in implant dentistry over 50 years (SR/MA, systematic review/meta analysis; $C T$, clinical trial; $C R$, case report; $R C T$, randomized clinical trial; $\mathrm{CS}$, cross sectional; $\mathrm{CC}$, case control; $\mathrm{COH}$, cohort)

\section{Discussion}

Over 5,500 articles are published every day in the medical literature, including the field of dentistry. ${ }^{9}$ The application of EBD requires that scholars and clinicians alike find and apply the conclusions of highquality evidence from a huge amount of available data to improve clinical decision-making. ${ }^{1,2}$ Although dentists are progressively being anticipated to use this concept, it remains uncertain whether high-quality data are being applied in daily clinical practice. ${ }^{1,2,10,11}$

Our results indicate that, overall, the type of studies in the dental literature has improved significantly over the last 50 years, with a pattern in the direction of publishing a higher frequency of randomized CTs and SRs/MAs. This improvement in evidence parallels an increasing number of articles that have been published recently.

There are several obstacles and challenges related to the application of EBD in dental practice. ${ }^{12,13}$ The ability of dentists, dental authorities, and other organizations to apply EBD in daily practice is questionable. There should be a greater effort by everyone to adopt the principles of EBD in everyday clinical practice. Moreover, dentists must be able to update themselves through their ability to access high-quality research that will help them in clinical decision-making.

Unfortunately, dental articles have faced criticism due to insufficient or incorrect methodologies, errors in statistical analyses, and demonstration of low quality overall like other medical fields as Slim et al. reported in surgery. ${ }^{13}$ These findings have been documented by several researchers who have noted a lack of well-designed studies in the dental field. ${ }^{14}$ For example, authors of several observational studies reported issues with the control group and bias. Further, Hall et al. found that various RCTs contained serious flaws including errors with randomization, blinding, sample size, allocation, concealment assessment of study endpoints, and results interpretation. ${ }^{15}$ Moreover, several articles have shown that the majority of negative results of RCTs lacked sufficient statistical power to find a significant difference among groups. ${ }^{16-19}$ Consequently, several dentists may be resistant toward EBD due to a lack of high-quality evidence. ${ }^{10,11}$

Regardless of the general problems associated with the quality of the dental literature, several attempts have been made to improve the research quality among dentists as reported in Manfredini et al. ${ }^{20}$ This fact is demonstrated by the number of articles related to statistics and epidemiology in the dental literature, as well as dental organizations, meetings, and courses dedicated solely to clinical research. Further, some dentists today are pursuing advanced training in clinical research, biostatistics, and/or epidemiology. ${ }^{20,21} \mathrm{~A}$ growing number of dental schools, academic departments, and graduate programs may require research department-most frequently in collaboration with statisticians and non-dental investigators. Most of the journals with a higher impact factor now have a statistical advisor serving on the board. As a result, we see recent studies with greater statistical complexity of clinical research and a related increase over time. $^{22}$

This study has several limitations. First, although a large percentage of higher hierarchical studies have been published, we do not know about the quality of these articles which will be a complicated task because the selected research types/designs are not one dimensional in terms of the amount of evidence they include. However, we will establish how the different research designs are ranked on an "evidence" scale in the future. The bibliometric analysis selected only 33 journals to evaluate dental literature. This process excluded several other journals, so there may be other articles that illuminate other trends that were missed. It was also difficult to mask reviewers to journal name and the year of publication when assessing the articles. Although this potentially introduced some observer bias, all investigated variables were clear and easy to assess (e.g., "study design" and "main topic," etc.), thus, reducing this possibility of bias. Finally, the results of this study are applicable to the included journals and do not represent all dental journals. However, these journals are could be considered representative of the available evidence currently being used in daily clinical practice. ${ }^{23}$

\section{Conclusion}

Our analysis of the published dental research reveals that the methodology of dental research designs has been significantly enhanced over the last 50 years. The data suggest that dental researchers perhaps need further methodological training to publish better quality evidence, which has implications in EBD and dental practice overall.

\section{Clinical Significance}

Types of studies used in the dental field are not yet investigated. Thus, little is known about the common study design types in dental literature. This can affect the decision made regarding technique, risk factors, prevention, or treatment.

\section{Availability of Data and Material}

All co-authors agree to disclose publicly for all available datasets presented in the main paper.

\section{Authors' Contributions}

Conceived and designed the study: ZN. Literature search, study inclusion, and data extraction: $Z N, A A, A S, E N, H A, L S, M N, R B, R Q$, SW, SA, and YM. Analyzed the data: ZN, AA, AS, EN, HA, LS, MN, RB, $R Q, S W, S A$, and $Y M$. Prepared the manuscript: $Z N, A A, A S, E N, H A$, $\mathrm{LS}, \mathrm{MN}, \mathrm{RB}, \mathrm{RQ}, \mathrm{SW}, \mathrm{SA}$, and $\mathrm{YM}$. All authors read and approved the final manuscript. 


\section{References}

1. El-Rabbany M, Li S, et al. A Quality Analysis of Systematic Reviews in Dentistry, Part 1: Meta-Analyses of Randomized Controlled Trials. J Evid Based Dent Pract 2017;17:389-398. DOI: 10.1016/ j.jebdp.2017.06.004.

2. Frantsve-Hawley J, Jeske A. The American Dental Association's Center for Evidence-Based Dentistry: a critical resource for 21st century dental practice. Tex Dent J 2011;128:201-205.

3. Gopalakrishnan S, Ganeshkumar P. Systematic Reviews and Metaanalysis: Understanding the Best Evidence in Primary Healthcare. J Family Med Prim Care 2013;2:9-14. DOI: 10.4103/2249-4863.109934.

4. Kranke P. Evidence-based practice: how to perform and use systematic reviews for clinical decision-making. Eur J Anaesthesiol 2010;27:763-772. DOI: 10.1097/EJA.0b013e32833a560a.

5. Evans D. Hierarchy of evidence: a framework for ranking evidence evaluating healthcare interventions. J Clin Nurs 2003;12:77-84. DOI: 10.1046/j.1365-2702.2003.00662.x.

6. Lau J, loannidis JP, et al. Summing up evidence: one answer is not always enough. Lancet 1998;351:123-127. DOI: 10.1016/S01406736(97)08468-7.

7. Egger M, Smith GD, et al. Meta-analysis: principles and procedures. BMJ 1997;315:1533-1537. DOI: 10.1136/bmj.315.7121.1533.

8. Guyatt GH, Haynes RB, et al. Users' Guides to the Medical Literature: XXV. Evidence-based medicine: principles for applying the Users' Guides to patient care. Evidence-Based Medicine Working Group. JAMA 2000;284:1290-1296. DOI: 10.1001/jama.284.10.1290.

9. Liumbruno GM, Velati C, et al. How to write a scientific manuscript for publication. Blood Transfus 2013;11:217-226. DOI: 10.2450/2012. 0247-12.

10. Brignardello-Petersen R, Carrasco-Labra A, et al. A practical approach to evidence-based dentistry: understanding and applying the principles of EBD. J Am Dent Assoc 2014;145:1105-1107. DOI: 10.14219/ jada.2014.102.
11. Ismail Al, Bader JD. Evidence-based dentistry in clinical practice. J Am Dent Assoc 2004;135:78-83. DOI: 10.14219/jada.archive.2004.0024.

12. Maier RV. What the surgeon of tomorrow needs to know about evidence-based surgery. Arch Surg 2006;141:317-323. DOI: 10.1001/ archsurg.141.3.317.

13. Slim K. Limits of evidence-based surgery. World J Surg 2005;29:606609. DOI: 10.1007/s00268-005-7922-x.

14. Hall JC, Mills B, et al. Methodologic standards in surgical trials. Surgery 1996;119:466-472. DOI: 10.1016/S0039-6060(96)80149-8.

15. Hall JC, Hall JL. Randomization in surgical trials. Surgery 2002;132:513518. DOI: $10.1067 / \mathrm{msy} .2002 .125350$.

16. Chang DC, Matsen SL, et al. Why should surgeons care about clinical research methodology? J Am Coll Surg 2006;203:827-830. DOI: 10.1016/j.jamcollsurg.2006.08.013.

17. Dimick JB, Diener-West $M$, et al. Negative results of randomized clinical trials published in the surgical literature: equivalency or error? Arch Surg 2001;136:796-800. DOI: 10.1001/archsurg.136.7.796.

18. Maggard MA, O'Connell JB, et al. Sample size calculations in surgery: are they done correctly? Surgery 2003;134:275-279. DOI: 10.1067/ msy.2003.235.

19. Manterola C, Pineda V, et al. What is the methodologic quality of human therapy studies in ISI surgical publications? Ann Surg 2006:244:827-832. DOI: 10.1097/01.sla.0000242708.51631.66.

20. Manfredini D, GreeneCS, et al. Evidence-based dentistry or meta-analysis illness? A commentary on current publishing trends in the field of temporomandibular disorders and bruxism. J Oral Rehabil 2019;46:1-4.

21. Activities TACOSE. The American College of Surgeons Educational Activities.

22. Kurichi JE, Sonnad SS. Statistical methods in the surgical literature. J Am Coll Surg 2006;202:476-484. DOI: 10.1016/j.jamcollsurg.2005.11.018.

23. Nieminen $P$, Carpenter J, et al. The relationship between quality of research and citation frequency. BMC Med Res Methodol 2006;6:42. DOI: 10.1186/1471-2288-6-42. 